\title{
First Clarity, Then Brevity
}

\author{
Khaled Moustafa
}

The optimization of scientific communication depends, at least in part, on the elimination of linguistic obstacles and ambiguous prose [1]. The ability to communicate scientific information effectively is an essential skill to have as a scientist but also as a tool to popularize scientific knowledge for the public [2]. Acronyms and abbreviations in scientific manuscripts may help attain some aspects in this important endeavor for example to avoid cumbersome repetitions, save space, and speed up the reading flow of a manuscript. However, the overuse of abbreviations and acronyms may also hamper the reading process and render the written text cryptic. As there is no strict guideline or obligation for authors to use or not to use acronyms, many authors are tempted to misuse abbreviations, including in titles and abstracts of their manuscripts, particularly when they try to introduce new ones. However, the use of abbreviations and acronyms in scientific writing is not mandatory or even necessary [ $\underline{3}$ ]. Although some journals ask authors to provide abbreviations' lists, such lists are often placed at the beginning of the main text, obliging readers to scroll up/down or to turn pages forward/backward many times to recall the meaning of an abbreviation they encounter far in the text. Repetitive scrolling or back/forth movements could thus overwhelm the reader, especially when the abbreviations are many, ambiguous or unfamiliar.

Scientific papers should not be boring [4] but the overuse of abbreviations and acronyms may render the reading of potentially worthwhile papers boring rather than appealing. In the same way that we need to teach more effectively [ㄷ], we also need to communicate written texts more efficiently without verbosity, vague or complex abbreviations.

To avoid the troublesome use of abbreviations, and to be effective and digestible, scientific manuscripts need to be written in a clear and direct style without ambiguous or complex abbreviations or acronyms. An easily comprehensible text should be preferable over an excessive use of uncommon abbreviations or acronyms. Journals may need to set up clear rules to limit the use of abbreviations that should be tolerated only if the abbreviation itself is short, easy to memorize, common, or if the full name is overly long. An effective science communication needs to be clear to be rewarding [6] . It might also be good to adopt a standardized terminology []]. To relay complex scientific concepts more efficiently, it would be useful to offer formal and consistent training early in the career of scientists to improve their communication skills with the public and with other non-specialist scientists [8]. It should also be unnecessary to create new equivocal abbreviations or acronyms for each new expression or 
concept introduced in new studies. Moreover, as publications are now produced electronically, space saving is no longer an issue to write full sentences rather than cryptic and uncommon abbreviations [3] . The use of uncommon acronyms excessively is indeed one of many other common practices that should be avoided in the scientific manuscripts []ㅡ. For the sake of good reading and comprehension of scientific papers, clarity should prevail on brevity. Much of the scientific information is already and inherently complex. The overuse of abbreviations and acronyms will add a new layer of complexity and convolution that could be easily avoided by simple attention and care to avoid enigmatic proses and abbreviations.

\section{References}

1. Drubin, D.G. and D.R. Kellogg, English as the universal language of science: opportunities and challenges. Molecular Biology of the Cell, 2012. 23(8): p. 1399-1399.

2. Goodwin, S.S., iBiology: communicating the process of science. Mol Biol Cell, 2014. 25(15): p. 2217-9.

3. Caon, M., Abbreviations, initialism and acronyms: their use in medical physics (THUMP). Australasian Physical \& Engineering Sciences in Medicine, 2016. 39(1): p. 11-12.

4. Chapman, P.M., Scientific papers should not be boring. Marine Pollution Bulletin, 2014. 87(1-2): p. 2.

5. Dolan, E.L. and J.P. Collins, We must teach more effectively: here are four ways to get started. Mol Biol Cell, 2015. 26(12): p. 2151-2155.

6. Irion, R., Science communication: a career where PhDs can make a difference. Mol Biol Cell, 2015. 26(4): p. 591-593.

7. Klionsky, D.J., Developing a set of guidelines for your research field: a practical approach. Mol Biol Cell, 2016. 27(5): p. 733-8.

8. Goldina, A. and O.I. Weeks, Science cafe course: an innovative means of improving communication skills of undergraduate biology majors. J Microbiol Biol Educ, 2014. 15(1): p. 13-7.

9. Caon, M., What should not be in a manuscript submitted to a scientific journal. Australasian Physical \& Engineering Sciences in Medicine, 2015. 38(2): p. 203-204.

\footnotetext{
'Email: khaled.moustafa@frenxiv.org
} 